\title{
Smad3 signaling activates bone marrow-derived fibroblasts in renal fibrosis
}

\author{
Jiyuan Chen ${ }^{1}$, Yunfeng Xia ${ }^{1}$, Xia Lin'², Xin-Hua Feng ${ }^{2}$ and Yanlin Wang ${ }^{1,3}$
}

Recent studies have demonstrated that bone marrow-derived fibroblasts contribute significantly to the pathogenesis of renal fibrosis. However, the signaling mechanisms underlying the activation of bone marrow-derived fibroblasts in the kidney are incompletely understood. As TGF- $\beta 1 / \mathrm{Smad} 3$ signaling has been shown to have an important role in the pathogenesis of kidney fibrosis, we investigated the role of Smad3 in the activation of bone marrow-derived fibroblasts in the kidney following obstructive injury using Smad3-knockout mice and Smad3-null monocytes. Compared with wildtype mice, Smad3-knockout mice accumulated significantly fewer bone marrow-derived fibroblasts in the kidney after obstructive injury. Furthermore, Smad3-knockout mice exhibited less myofibroblast activation and expressed less $\alpha$-SMA in the obstructed kidney. Consistent with these findings, genetic deletion of Smad3 reduced total collagen deposition and suppressed the expression of extracellular matrix proteins. Moreover, wild-type mice engrafted with Smad3 ${ }^{-1-}$ bone marrow cells displayed fewer bone marrow-derived fibroblasts in the kidney with obstructive injury and showed less severe renal fibrosis compared with wild-type mice engrafted with Smad3 ${ }^{+/+}$bone marrow cells. In cultured monocytes, TGF- $\beta 1$ induced phosphorylation of Smad3 and Smad3 deficiency abolished TGF- $\beta 1$-induced expression of $\alpha$-SMA and extracellular matrix proteins. Taken together, our results demonstrate that Smad3 signaling has an essential role in the activation of bone marrow-derived fibroblasts in the kidney during the pathogenesis of renal fibrosis.

Laboratory Investigation (2014) 94, 545-556; doi:10.1038/labinvest.2014.43; published online 10 March 2014

KEYWORDS: bone marrow-derived cells; chronic kidney disease; cytokine; extracellular matrix; fibroblasts; renal fibrosis

Renal fibrosis is a hallmark of chronic kidney disease regardless of the underlying etiologies. ${ }^{1,2}$ Furthermore, interstitial fibrosis is a key structural component of obstructive nephropathy, which is the major cause of chronic kidney disease in children. ${ }^{3}$ Renal interstitial fibrosis is characterized by fibroblast activation and excessive production and deposition of extracellular matrix (ECM), which causes the destruction and collapse of renal parenchyma and progressive loss of kidney function. Because activated fibroblasts are the principal cells responsible for ECM production, their activation is regarded as a key event in the pathogenesis of renal fibrosis. ${ }^{4,5}$ Recent evidence indicates that these cells may originate from bone marrowderived fibroblast progenitor cells. ${ }^{6-11}$

Bone marrow-derived fibroblasts termed fibrocytes are derived from a subpopulation of monocytes via monocyte- to-fibroblast transition. ${ }^{12-16}$ These cells express mesenchymal markers such as collagen I, vimentin, and discoid domain receptor 2 (DDR2), and hematopoietic markers such as CD45 and CD11b. ${ }^{13,17-19}$ These cells in culture display an adherent, spindle-shaped morphology and express $\alpha$-SMA, consistent with the concept that they can differentiate into myofibroblasts. ${ }^{17-19}$ We and others have shown that bone marrow-derived fibroblasts contribute significantly to the pathogenesis of renal fibrosis. ${ }^{5,8,14}$ However, the molecular mechanisms underlying the activation of these cells in the kidney are incompletely understood.

TGF- $\beta 1$ has a key role in the pathogenesis of renal fibrosis through the activation of a cascade of intracellular signaling pathways. $^{20-23}$ Evidence suggests that activation of the Smad signaling cascade is important in the regulation of ECM protein expression and tissue fibrosis. ${ }^{24-28}$ Although Smad3

\footnotetext{
${ }^{1}$ Division of Nephrology, Department of Medicine-Nephrology, Baylor College of Medicine, Houston, TX, USA; ${ }^{2}$ Michael E. DeBakey Department of Surgery and Department of Molecular and Cellular Biology, Baylor College of Medicine, Houston, TX, USA and ${ }^{3}$ Medical Care Line, Michael E DeBakey Veterans Affairs Medical Center, Houston, TX, USA

Correspondence: Dr Y Wang, MD, PhD, Division of Nephrology, Department of Medicine-Nephrology, Baylor College of Medicine, BCM395, One Baylor Plaza, Houston TX 77030, USA.

E-mail: yanlinw@bcm.edu

Received 21 September 2013; revised 3 January 2014; accepted 14 January 2014
} 
is essential for the development of renal fibrosis, the cellular and molecular mechanisms underlying its profibrotic actions are not fully understood.

In this study, we examined the role of Smad3 in the activation of bone marrow-derived fibroblasts in the kidney in a well-established model of tubulointerstitial fibrosis induced by unilateral ureteral obstruction (UUO) using Smad3knockout $(\mathrm{KO})$ mice and Smad3 $3^{-1-}$ monocytes. Our results demonstrate that genetic disruption of Smad3 prevents the development of renal fibrosis by suppressing the activation of $\mathrm{CD} 45^{+}$myeloid fibroblasts.

\section{MATERIALS AND METHODS Animals}

Animal experiments were approved by the Institutional Animal Care and Use Committee of Baylor College of Medicine (IACUC permit no.: AN-5011). The investigation conforms with the recommendations in the Guide for the Care and Use of Laboratory Animals published by the US National Institutes of Health (NIH Publication No. 85-23, revised 1996). All efforts were made to minimize suffering. The Smad3-KO mice on a background of C57BL6/J were kindly provided by Dr Xiao-Fan Wang. ${ }^{29}$ Smad3-KO mice and WT littermates at 8-12 weeks old were subjected to UUO surgery as described. ${ }^{11,12,30,31}$ Mice were allowed to recover from anesthesia and were housed in standard rodent cages with ad libitum access to water and food until they were killed.

\section{Renal Morphology}

Mice were killed and perfused by injections of PBS into the left ventricle of the heart to remove blood. One portion of the renal tissue was fixed in $10 \%$ buffered formalin and embedded in paraffin, cut at $5 \mu \mathrm{m}$ thickness, and stained with hematoxylin and eosin for initial evaluation, or picrosirius red to identify collagen fibers. The picrosirius red-stained sections were scanned using a microscope equipped with a digital camera (Nikon, Melville, NY) and quantitative evaluation was performed using the NIS-Elements $\mathrm{Br} 3.0$ software as described..$^{11}$ The collagen-stained area was calculated as a percentage of the total area.

\section{Quantitative Real-Time RT-PCR}

Quantitative analysis of the target mRNA expression was performed with real-time reverse transcription-polymerase chain reaction (RT-PCR) by the relative standard curve method. Total RNA was extracted from snap-frozen kidney tissues with TRIzol Reagent (Invitrogen). Aliquots $(1 \mu \mathrm{g})$ of total RNA were reverse-transcribed and amplified in triplicate using IQ SYBR Green Supermix reagent (Bio-Rad, Hercules, CA) with a real-time PCR machine (Bio-Rad), according to the manufacturer's instructions. The specificity of real-time PCR was confirmed by melting-curve analysis. The expression levels of the target genes were normalized to the GAPDH level in each sample. The following are the primer sequences: collagen I (forward, 5'-TGCCGCGACCTCAAGATGTG-3' and reverse, 5'-CACAAGGGTGCTGTAGGTGA-3'); fibronectin (forward, $5^{\prime}$-CTTCTCCGTGGAGTTTTACCG- $3^{\prime}$ and reverse, $\quad 5^{\prime}$-GCTGTCAAATTGAATGGTGGTG- $3^{\prime}$ ); $\alpha$-SMA (forward, $5^{\prime}$-ACTGGGACGACATGGAAAAG- $3^{\prime}$ and reverse, 5'-CATCTCCAGAGTCCAGCACA-3'); GAPDH (forward, $5^{\prime}$ TGCTGAGTATGTCGTGGAGTCTA- $3^{\prime}$ and reverse, $5^{\prime}$-AGTG GGAGTTGCTGTTGAAATC-3').

\section{Immunofluorescence}

Renal tissues were embedded in OCT compound, snapfrozen on dry ice, cut at $5 \mu \mathrm{m}$ thickness, and mounted on microscope slides. After fixation, nonspecific binding was blocked with serum-free protein block (Dako). Sections were then incubated with rabbit anti-phospho-Smad3 antibody (Rockland) followed by Alexa-488-conjugated donkey antirabbit antibody (Invitrogen), rabbit anti-collagen I antibody (Rockland), followed by Alexa-488-conjugated donkey antirabbit antibody (Invitrogen), rabbit anti-fibronectin antibody (Sigma), followed by Alexa-488-conjugated donkey anti-rabbit antibody (Invitrogen), or rabbit anti- $\alpha$-SMA antibody (Abcam), followed by Alexa-488-conjugated donkey anti-rabbit antibody (Invitrogen). For double immunofluorescence, renal tissues were fixed and stained with primary antibodies, followed by appropriate secondary antibodies sequentially. Slides were mounted with mounting medium containing DAPI. Fluorescence intensity was visualized using a microscope equipped with a digital camera (Nikon, Melville, NY). Quantitative evaluation of sections stained for $\alpha$-SMA was performed using the NIS-Elements $\mathrm{Br}$ 3.0 software. The fluorescence-positive area was calculated as a percentage of the total area.

\section{Cell Isolation and Flow Cytometry}

Renal cell isolation and flow cytometry were performed as described. ${ }^{11}$ Briefly, kidneys were minced and incubated at $37^{\circ} \mathrm{C}$ for $30 \mathrm{~min}$ in PBS containing $0.25 \mathrm{mg} / \mathrm{ml}$ Liberase TM (Roche) and $10 \mathrm{U} / \mathrm{ml}$ DNase (Roche). Cells were filtered through a $40 \mu \mathrm{m}$ strainer, washed, centrifuged, and resuspended in FACS buffer. Cells $\left(5 \times 10^{5}\right)$ were first incubated with FITC-anti-CD45 (BD Biosciences, San Jose, CA), and then goat-anti-DDR2 (Santa Cruz Biotech, Santa Cruz, CA), followed by followed by anti-goat-APC (BD Biosciences). Cells incubated with irrelevant isotype-matched antibodies (BD Biosciences) and unstained cells were used as controls. The cutoffs were set according to results of controls. The fluorescence intensities were measured using a BD LSR II flow cytometer (BD Biosciences). Data were analyzed using the BD FACSDiva software.

\section{Western Blot Analysis}

Protein was extracted using the RIPA buffer containing a cocktail of proteinase inhibitors (Thermo Fisher Scientific, Rockford, IL) and quantified with Bio-Rad protein assay. Equal amounts of protein were separated on SDS-polyacrylamide gels in a Tris/glycine buffer system, transferred 
onto nitrocellulose membranes, and blotted according to standard procedures with primary antibodies (phosphoSmad3, collagen I, fibronectin, and $\alpha$-SMA). Membranes were then stripped and reblotted with anti-GAPDH antibody (Millipore, Billerica, CA). The specific bands of target proteins were analyzed using an Odyssey IR scanner and band intensities were quantified using NIH Image/J.

\section{Bone Marrow Transplantation}

Bone marrow transplantation was performed as described previously. ${ }^{11}$ Briefly, bone marrow cells $\left(5 \times 10^{6}\right)$ from WT or Smad3-KO mice were transferred to lethally irradiated WT C57BL/6 mice. After transplantation, mice were allowed to recuperate for 2 months before induction of kidney injury by UUO.

\section{Monocyte Isolation and Culture}

Monocytes from spleen of WT and Smad3-KO mice were isolated as described. ${ }^{32}$ Monocytes were cultured in RPMI 1640 medium containing 10\% FBS, $1 \%$ penicillin and streptomycin in an atmosphere of $5 \% \mathrm{CO}_{2}$, and $95 \%$ air at $37^{\circ} \mathrm{C}$. For TGF- $\beta 1$ treatment, cells were starved for $24 \mathrm{~h}$ by incubation with RPMI 1640 containing $1 \%$ FBS and then exposed to vehicle or TGF- $\beta 1$ at $10 \mathrm{ng} / \mathrm{ml}$ for $24 \mathrm{~h}$.

\section{Statistical Analysis}

All data were expressed as mean \pm s.e.m. Multiple group comparisons were performed by one-way ANOVA, followed by the Bonferroni procedure for comparison of means. Comparisons between two groups were analyzed by the two-tailed Student's $t$-test. $P<0.05$ was considered statistically significant.

\section{RESULTS}

\section{Smad3 is Activated in CD45 ${ }^{+}$Myeloid Fibroblasts during Renal Fibrosis}

We first characterized the activation of Smad3 in the kidney in a mouse model of tubulointerstitial fibrosis induced by UUO. Kidney sections were stained with phospho-Smad3 antibody and examined with a fluorescence microscope. Phospho-Smad3-positive signal was primarily detected in the interstitial cells of obstructed kidney (Figure 1a). To determine if Smad3 was activated in fibroblasts, renal sections were stained for phospho-Smad3 and DDR2, a fibroblastspecific marker, and examined with a fluorescence microscope. DDR2-positive fibroblasts were stained positive for phospho-Smad3 (Figure 1b). To examine if Smad3 was activated in $\mathrm{CD}_{4}{ }^{+}$myeloid fibroblasts, freshly isolated renal cells were stained for CD45, collagen I, and phospho-Smad3. $\mathrm{CD}_{4}{ }^{+}$and collagen $\mathrm{I}^{+}$cells were stained positive for phospho-Smad3, indicating that Smad3 in bone marrowderived fibroblasts are activated (Figure 1c). Western blot analysis confirmed that Smad3 was activated in the kidney in response to obstructive injury (Figures $1, \mathrm{~d}$, and e).

\section{Smad3 Deficiency Impairs Myeloid Fibroblast Accumulation}

To examine if Smad3 has a role in the accumulation of bone marrow-derived fibroblasts in the obstructed kidneys, WT and Smad3-KO mice were subjected to obstructive injury for 7 days. Kidney sections were stained for CD45 and procollagen I. Our results showed that the accumulation of $\mathrm{CD}_{4} 5^{+}$and procollagen $\mathrm{I}^{+}$fibroblasts was markedly increased in injured kidneys of WT mice, whereas the accumulation of $\mathrm{CD}_{4} 5^{+}$and procollagen $\mathrm{I}^{+}$fibroblasts was significantly reduced in injured kidneys of Smad3-KO mice (Figures 2a and b). Consistent with immunofluorescence staining, flow cytometric analysis of freshly isolated renal cells stained for CD45 and DDR2 demonstrated that Smad3 deficiency inhibited $\mathrm{CD}_{4} 5^{+}$and $\mathrm{DDR} 2^{+}$fibroblast accumulation in the kidney compared with WT mice (Figures $2 \mathrm{c}$ and $\mathrm{d}$ ). These data indicate that $\mathrm{Smad} 3$ has a role in the accumulation of bone marrow-derived fibroblasts in the kidney in response to obstructive injury.

\section{Smad3 Deficiency Inhibits Myofibroblast Activation}

As TGF- $\beta 1$ signaling has been shown to promote maturation of bone marrow-derived fibroblasts in vitro, ${ }^{19,33}$ we determined if Smad3 deficiency influences the development of myofibroblasts in the kidney. WT and Smad3-KO mice were subjected to UUO for 14 days. Kidney sections were stained with an antibody against $\alpha$-SMA, a marker of myofibroblasts, and examined with a fluorescence microscope. The results revealed that genetic deletion of Smad3 resulted in a significant reduction in the number of myofibroblasts in obstructed kidneys compared with WT mice (Figures 3a and b). Consistent with these findings, real-time RT-PCR showed that Smad3 deficiency significantly reduced the mRNA expression of $\alpha$-SMA compared with the level in WT mice with UUO (Figure 3c). These findings were confirmed at the protein levels by western blot analysis, which showed that Smad3 deficiency significantly reduced the protein expression levels of $\alpha$-SMA in obstructed kidneys compared with WT mice (Figures $3 \mathrm{~d}$ and e). These results indicate that Smad3 signaling has an important role in myofibroblast activation in the kidney in response to obstructive injury.

\section{Smad3 Deficiency Suppresses Renal Fibrosis}

As Smad3 regulates the accumulation of bone marrowderived fibroblasts in the kidney in response to obstructive injury, we then examined the effect of Smad3 deficiency on the development of renal fibrosis. WT and Smad3-KO mice were subjected to UUO for 14 days. WT mice developed significant collagen deposition in obstructed kidneys as demonstrated by picrosirius red staining. In contrast, Smad3-KO mice displayed much less collagen deposition in the kidney in response to obstructive injury (Figures 4a and b). These data indicate that Smad3 has a critical role in the pathogenesis of renal fibrosis. 
a
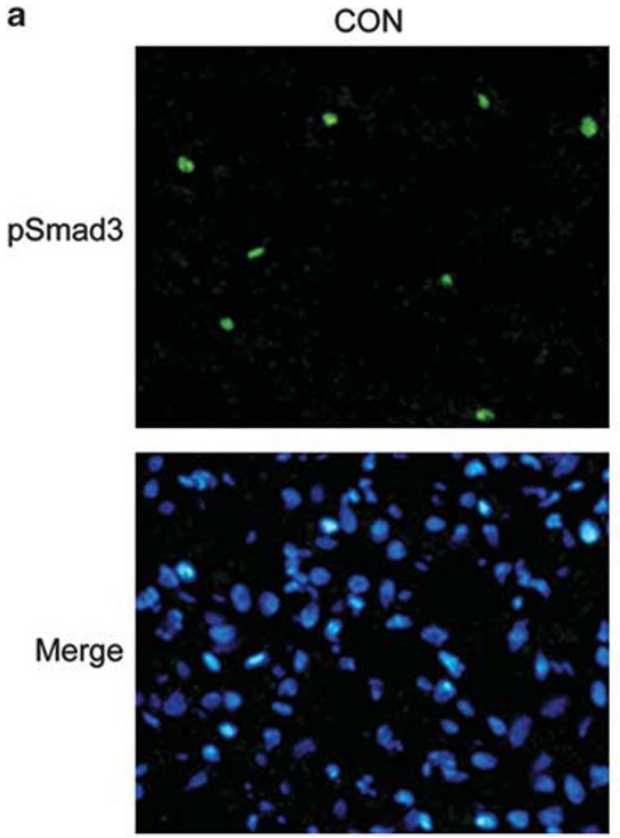

UUO
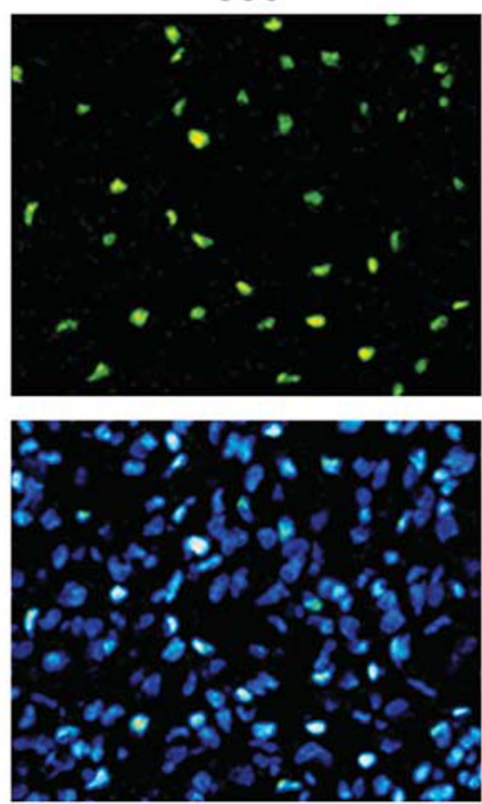

b
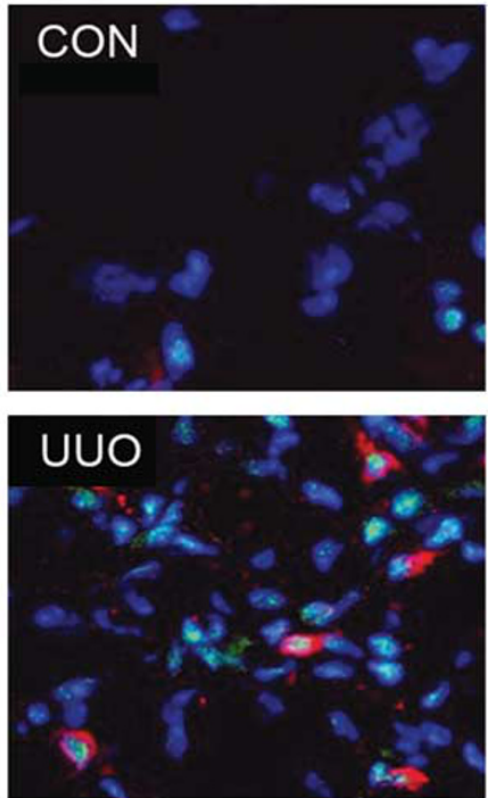

C
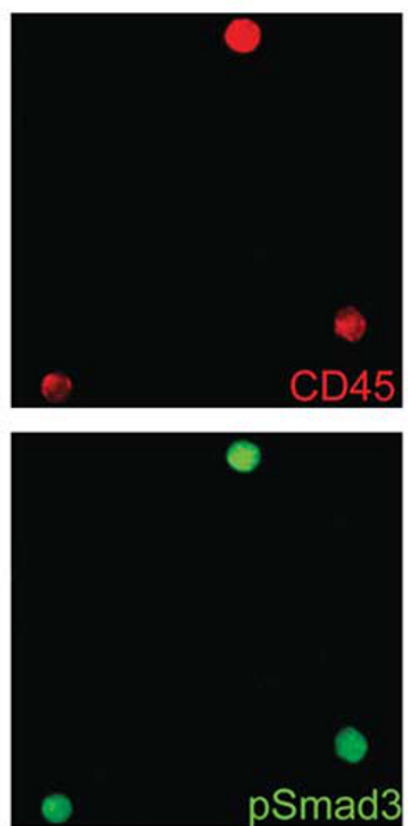
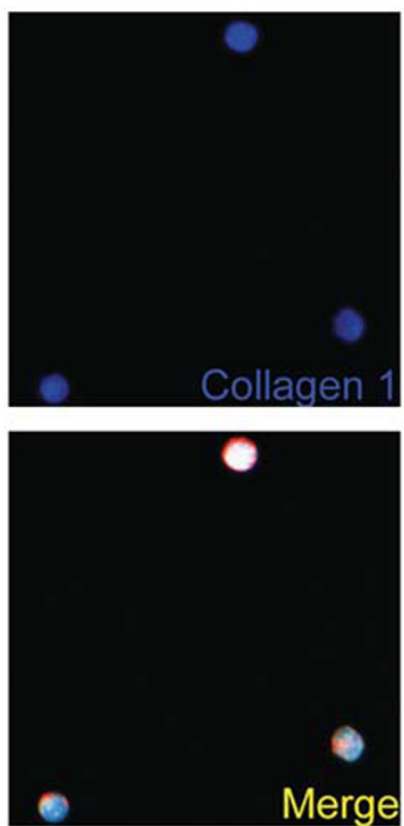

d
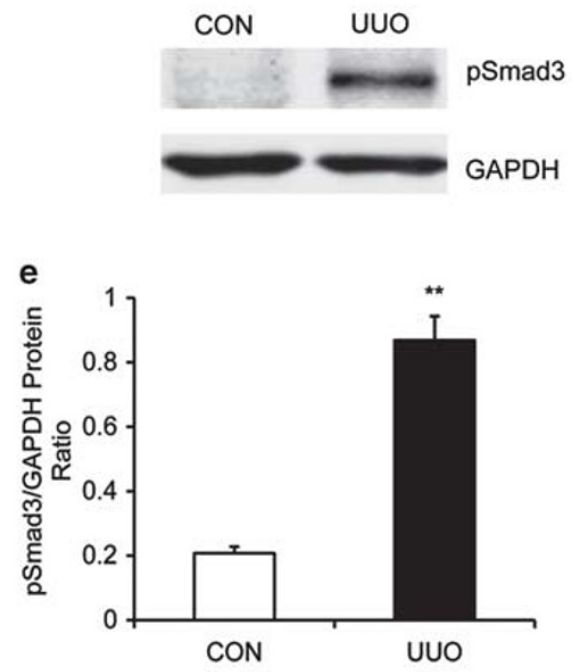

Figure $1 \mathrm{Smad} 3$ is activated in myeloid fibroblasts in the kidney after obstructive injury. (a) Representative photomicrographs of kidney sections stained for phospho-Smad3 (green) and counter stained with 4',6-diamidino-2-phenylindole (DAPI) (blue) (original magnification: $\times 400$ ).

(b) Representative photomicrographs of kidney sections stained for phospho-Smad3 (green) and discoid domain receptor 2 (DDR2) (red) and counter stained with DAPI (blue) (original magnification: $\times 600$ ). (c) Representative photomicrographs of isolated renal fibroblasts stained for CD45 (red), phospho-Smad3 (green), and collagen I (blue). (d) Representative western blot analysis show activation of Smad3 in unilateral ureteral obstruction (UUO) kidneys of wild-type (WT) mice. (e) Quantitative analysis of Smad3 phosphorylation in the control and UUO kidneys of WT mice. ${ }^{* *} P<0.01$ vs WT controls $(n=6)$.

We next investigated the effect of genetic disruption of Smad3 on the expression and accumulation of collagen I and fibronectin, two major components of ECM. There was a marked increase in the mRNA expression levels of collagen I and fibronectin in obstructed kidneys of WT mice, whereas genetic disruption of Smad3 significantly suppressed the mRNA expression levels of these matrix components in obstructed kidneys (Figures $5 \mathrm{a}$ and $\mathrm{b}$ ). We then examined ECM protein expression in the kidney in response to obstructive injury. Immunofluorescence staining revealed that genetic disruption of Smad3 inhibited collagen I and fibronectin protein expression in the obstructed kidneys compared with WT mice (Figures $5 \mathrm{c}$ and d). These results were confirmed by western blot analysis (Figures $5 \mathrm{e}-\mathrm{h}$ ). These data 
a
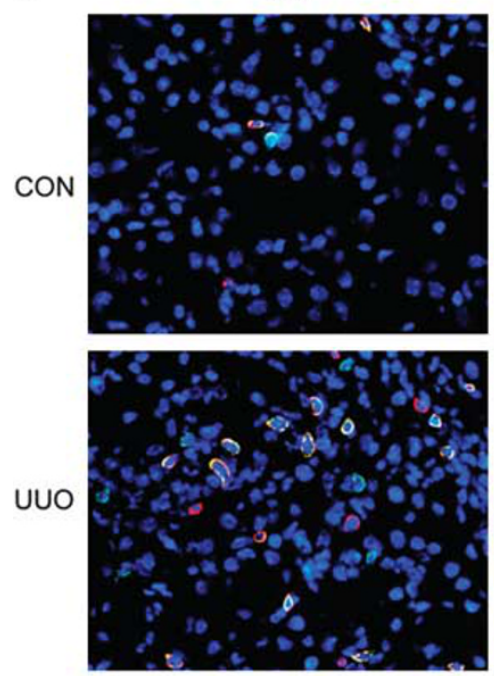

C

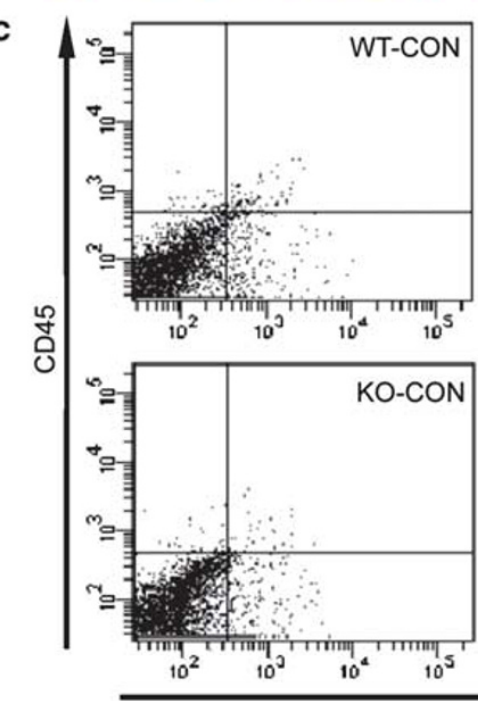

Smad3-KO
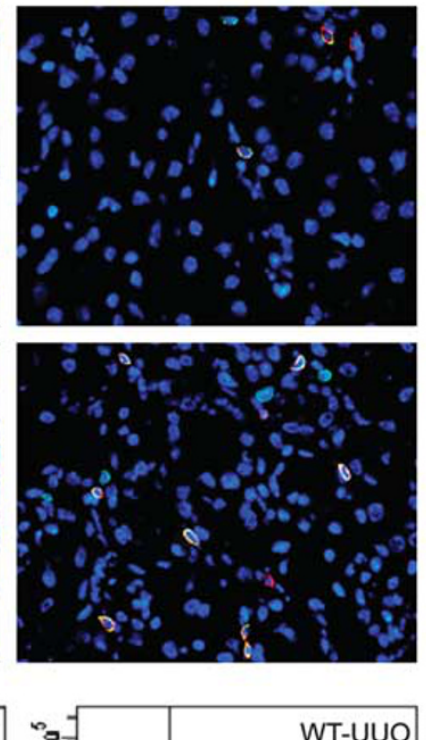
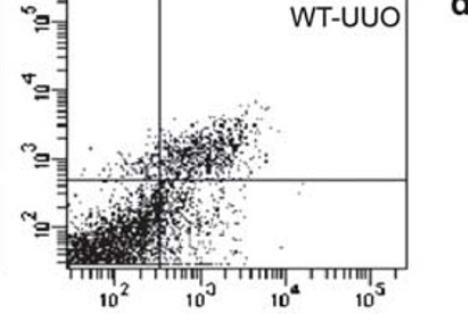

DDR2

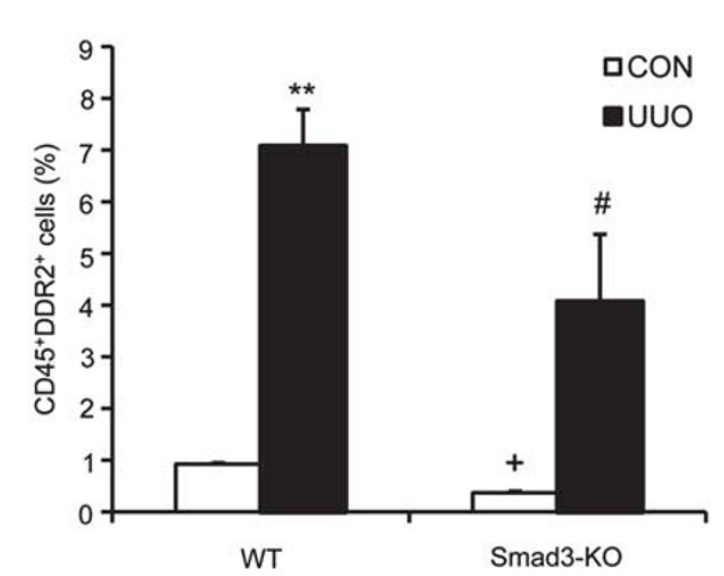

b

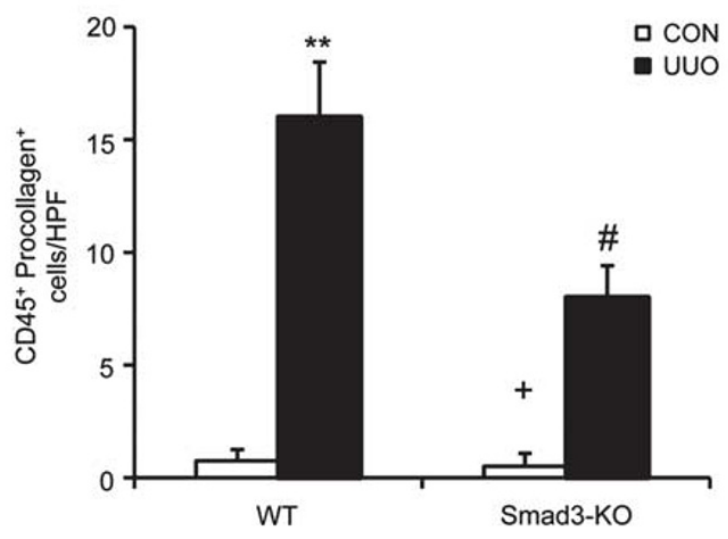

d

Figure 2 Smad3 deficiency suppresses the accumulation of bone marrow-derived fibroblasts in the kidney in response to obstructive injury. (a) Representative photomicrographs of kidney sections from wild-type (WT) and Smad3-knockout (KO) mice 1 week after unilateral ureteral obstruction (UUO) stained for CD45 (red), procollagen I (green), and 4',6-diamidino-2-phenylindole (DAPI) (blue). (b) Quantitative analysis of CD45 ${ }^{+}$and procollagen $\mathrm{I}^{+}$fibroblasts in the kidney of WT and Smad3-KO mice 1 week after unilateral ureteral obstruction (UUO). ${ }^{* *} P<0.01$ vs WT controls, ${ }^{+} P<0.05$ vs KO UUO and ${ }^{\#} P<0.05$ vs WT UUO ( $n=4$ per group). (c) Representative cytometric diagrams showing the effect of Smad 3 deficiency on the accumulation of CD45 and discoid domain receptor 2 (DDR2) dual-positive fibroblasts in the kidney in response to UUO. (d) Quantitative analysis of CD45 and DDR2 dualpositive fibroblasts in the kidney in response to UUO. ${ }^{* *} P<0.01$ vs WT control, ${ }^{+} P<0.05$ vs KO UUO, and ${ }^{\#} P<0.05$ vs WT UUO ( $n=3-4$ per group).

indicate that genetic deletion of Smad3 prevents renal fibrosis by inhibiting the production and deposition of ECM proteins.

\section{Smad3 Deficiency in Bone Marrow-derived Cells Reduces Renal Fibrosis}

To determine the role of Smad3 in bone marrow-derived cells in the development of renal fibrosis, we performed bone marrow transplant with $S$ mad3 $3^{+1+}$ or Smad $3^{-1-}$ bone marrow cells. Compared with WT mice transplanted with Smad3 ${ }^{+1+}$ bone marrow cells, WT mice transplanted with Smad3 ${ }^{-1-}$ bone marrow cells accumulated fewer bone marrow-derived fibroblasts (Figures $6 \mathrm{a}$ and $\mathrm{b}$ ) and showed a lesser degree of renal fibrosis (Figures $6 \mathrm{c}$ and d). These data indicate that myeloid Smad3 signaling is important for the activation of bone marrow-derived fibroblasts and the development of renal fibrosis.

\section{Smad3 Deficiency Inhibits Monocyte-to-Fibroblast Transition In Vitro}

To determine if Smad3 signaling regulates monocyte-tofibroblast transition in vitro, we treated monocytes from spleens of WT and Smad3-KO mice with TGF- $\beta 1$. 
a

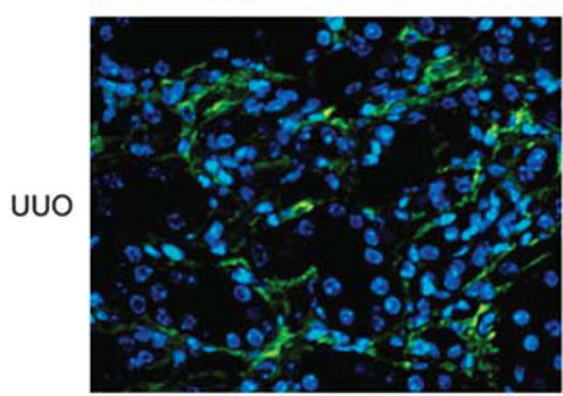

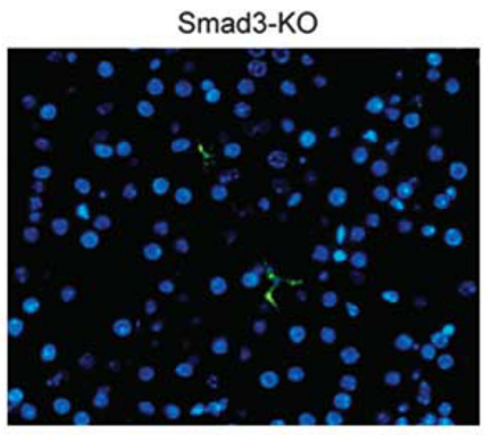

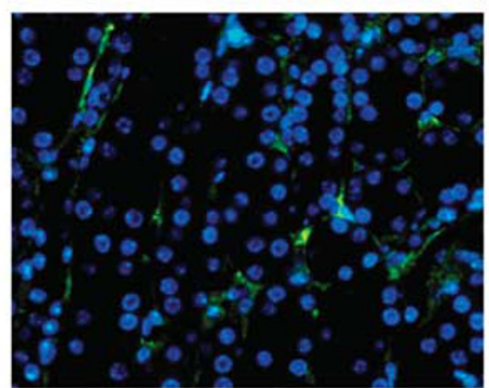

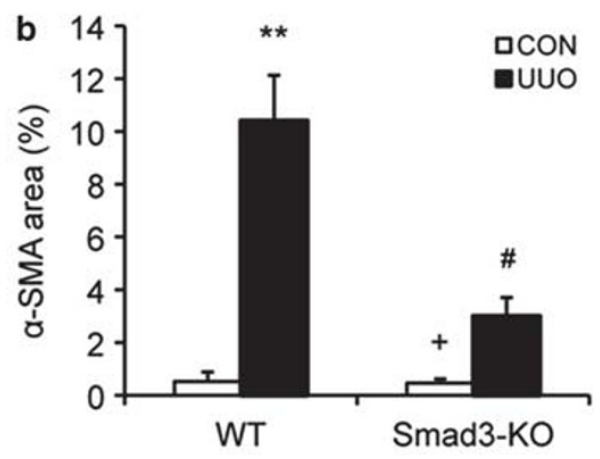

c

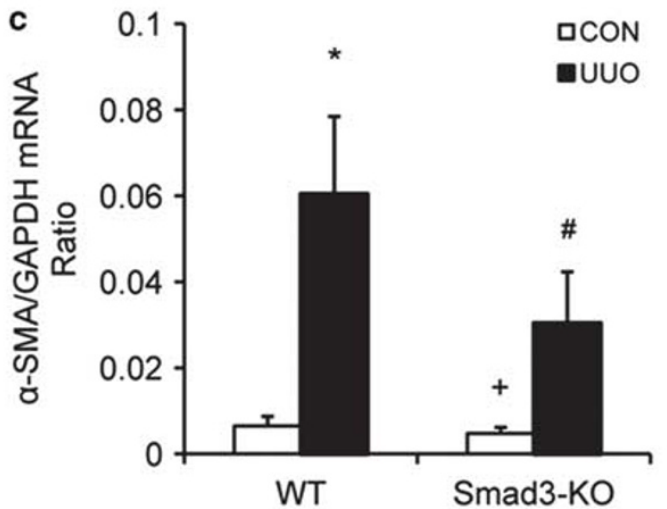

d
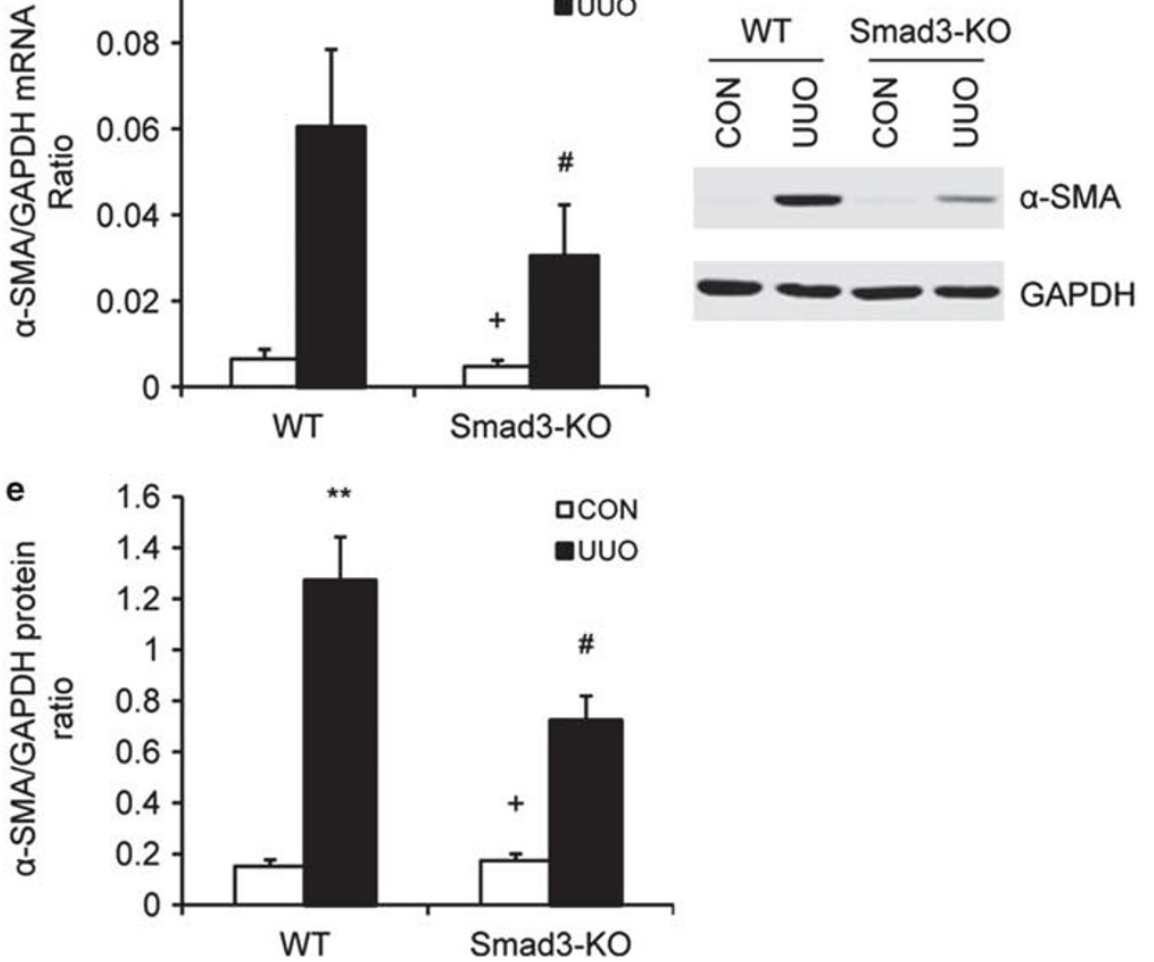

Figure 3 Smad3 deficiency inhibits myofibroblast activation and $\alpha$-smooth muscle actin ( $\alpha$-SMA) expression in obstructive nephropathy.

(a) Representative photomicrographs of $\alpha$-SMA immunofluorescence staining in the kidney of wild-type (WT) and Smad3-knockout (KO) mice 2 weeks after unilateral ureteral obstruction (UUO). (b) Quantitative analysis of $\alpha$-SMA protein expression in the kidney of WT and Smad3-KO mice 2 weeks after UUO. ${ }^{*} P<0.01$ vs WT controls, ${ }^{+} P<0.05$ vs KO UUO, and ${ }^{\#} P<0.05$ vs WT UUO ( $n=5$ per group). (c) The mRNA levels of $\alpha$-SMA in the kidney of WT and Smad3-KO mice as determined by real-time reverse transcription-polymerase chain reaction (RT-PCR). ${ }^{*} P<0.05$ vs WT controls, ${ }^{+} P<0.05$ vs KO UUO, and ${ }^{\#} P<0.05$ vs WT UUO ( $n=4$ per group). (d) Representative western blot analyses show the levels of $\alpha$-SMA protein expression in the kidney of WT and Smad3-KO mice. (e) Quantitative analysis of $\alpha$-SMA protein expression in the kidney of WT and Smad3-KO mice. ${ }^{* *} P<0.01$ vs WT controls, ${ }^{+} P<0.05$ vs KO UUO, and ${ }^{\#} P<0.05$ vs WT UUO ( $n=5$ per group). 
a
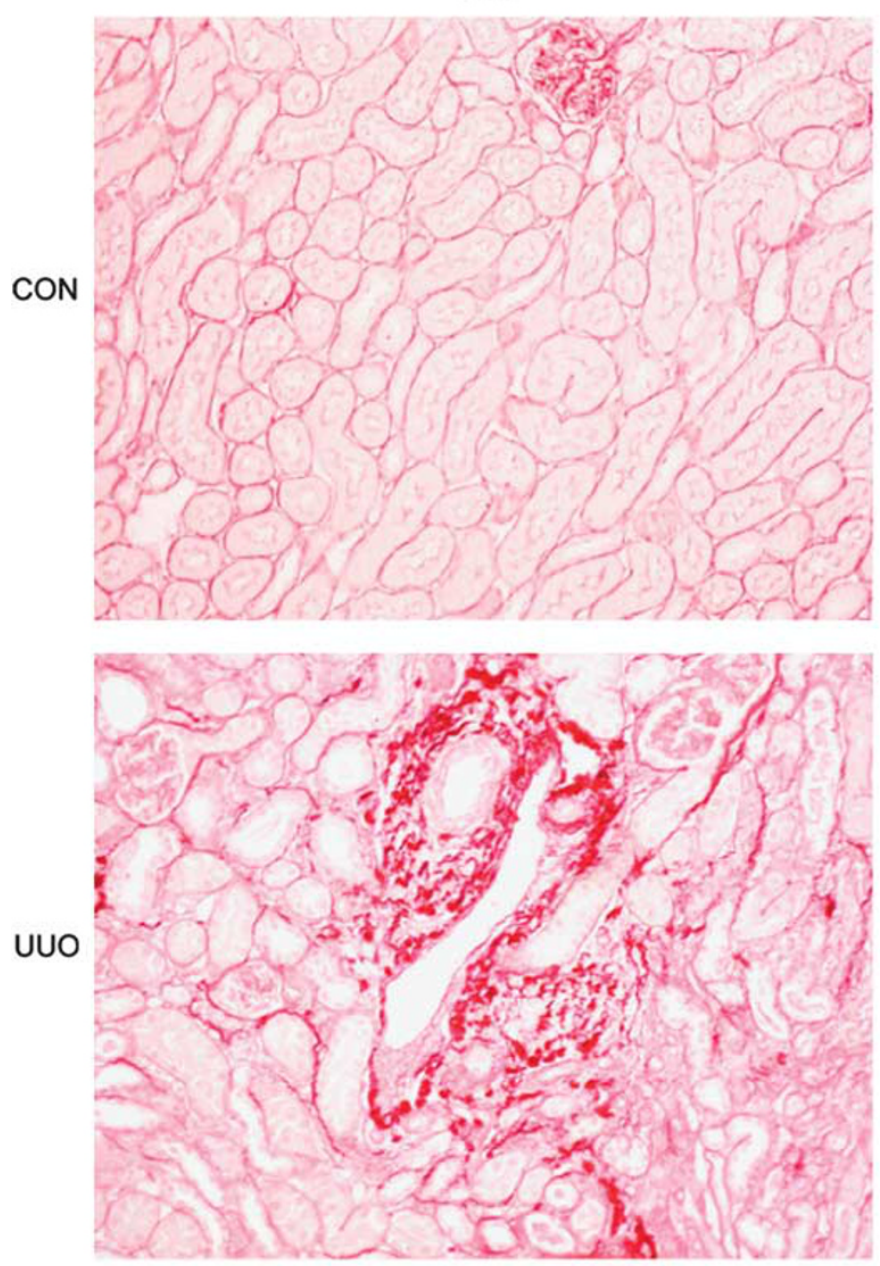

\section{Smad3-KO}
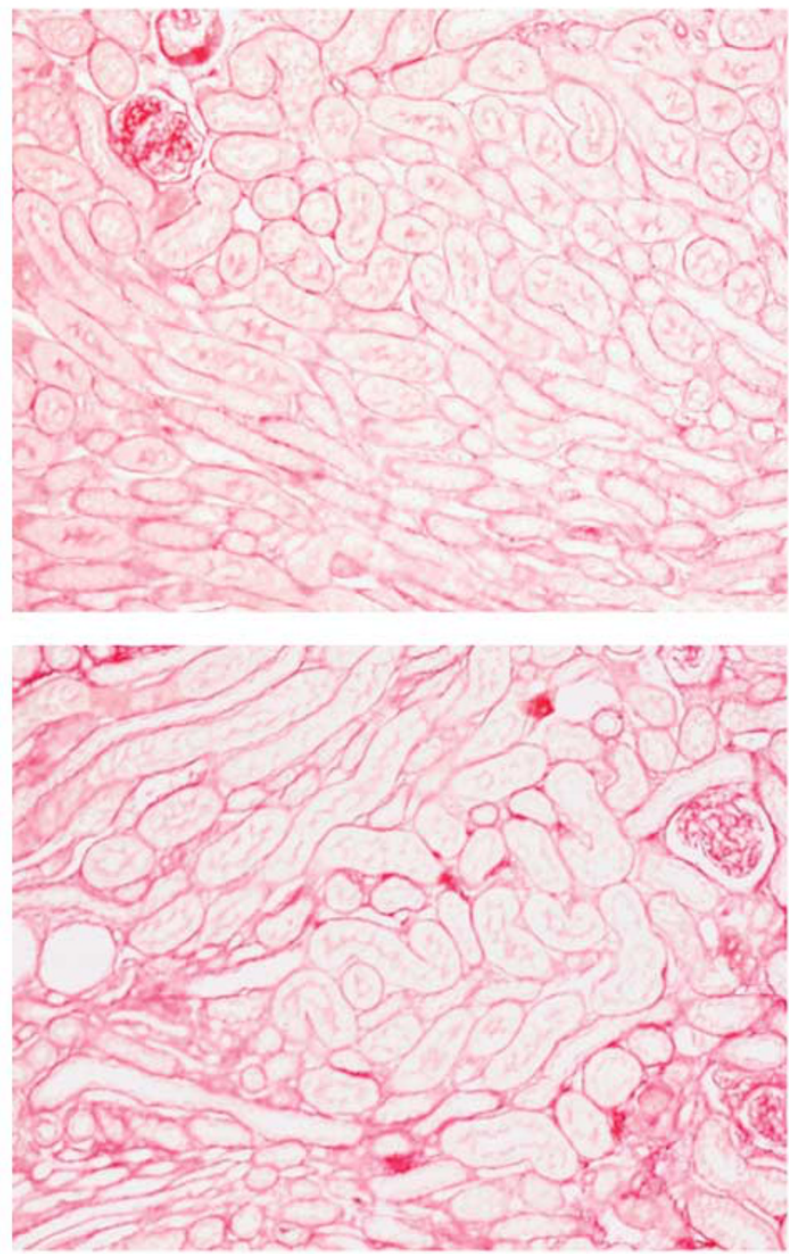

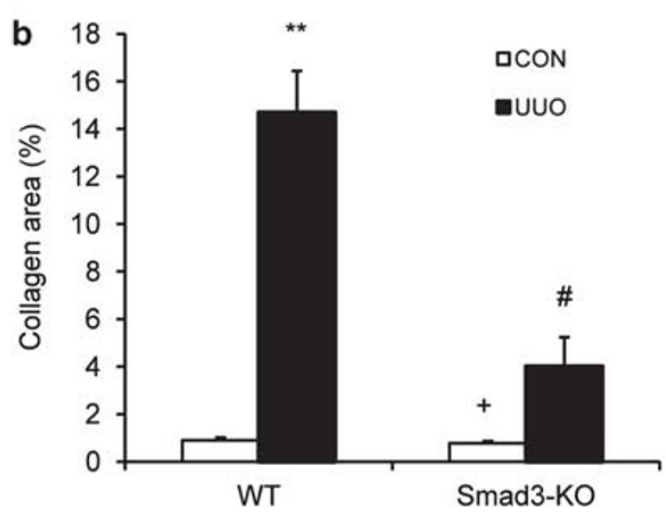

Figure 4 Smad3 deficiency suppresses renal fibrosis and collagen deposition in the kidney. (a) Representative photomicrographs show kidney sections stained with picrosirius red for assessment of total collagen deposition. (b) Quantitative analysis of renal interstitial collagen in different groups as indicated. ${ }^{* *} P<0.01$ vs wild-type (WT) controls, ${ }^{+} P<0.05$ vs knockout (KO) unilateral ureteral obstruction (UUO), and ${ }^{\#} P<0.05$ vs WT UUO ( $n=6$ per group).

Smad $3^{-1-}$ monocytes express less fibronectin and $\alpha$-SMA at basal conditions compared with Smad $3^{+1+}$ monocytes. Treatment of Smad3 ${ }^{+1+}$ monocytes with TGF- $\beta 1$ induced phosphorylation of Smad3 as well as expression of collagen I, fibronectin, and $\alpha$-SMA. In contrast, treatment of
Smad $3^{-1-}$ monocytes with TGF- $\beta 1$ did not induce expression of collagen I, fibronectin, and $\alpha$-SMA (Figure 7). These data support our in vivo observations that Smad3 signaling has an important role in the activation of bone marrowderived fibroblasts and pathogenesis of renal fibrosis. 

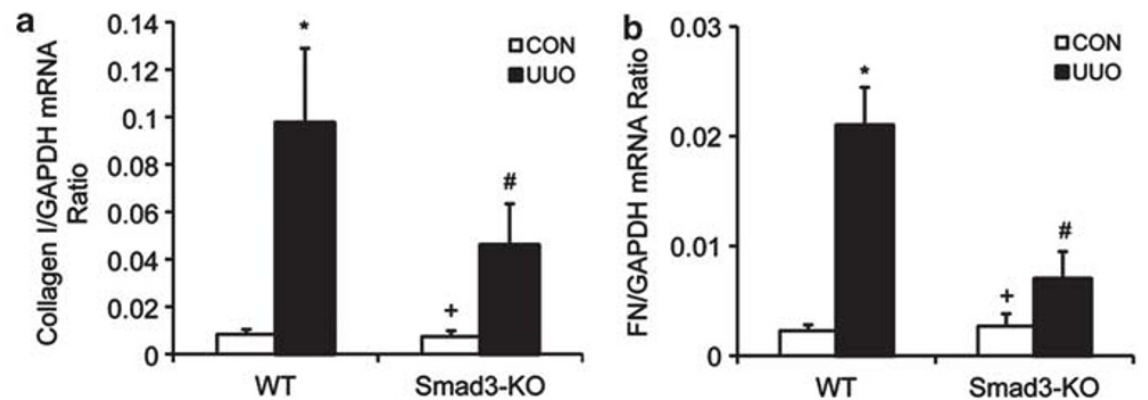

C
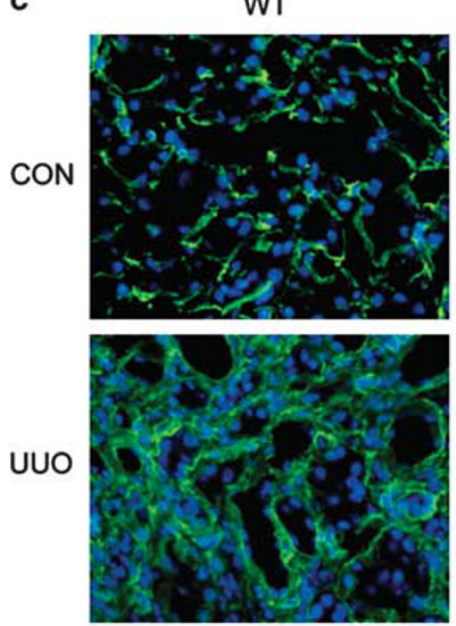
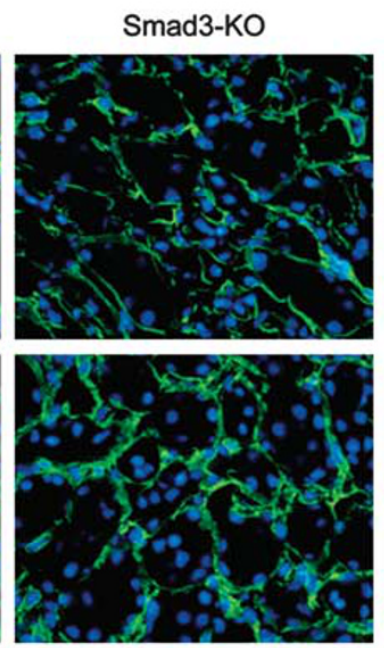

d
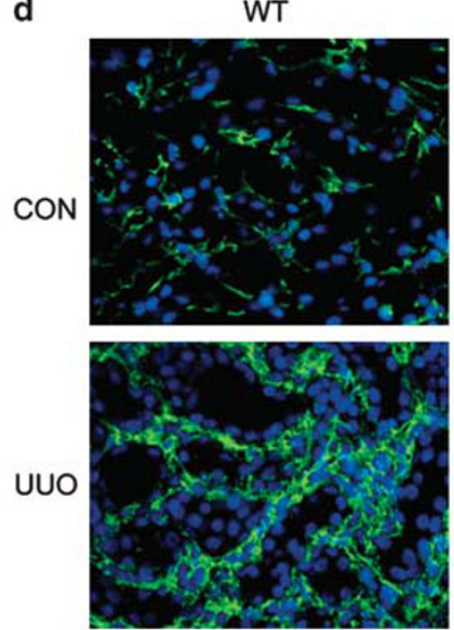
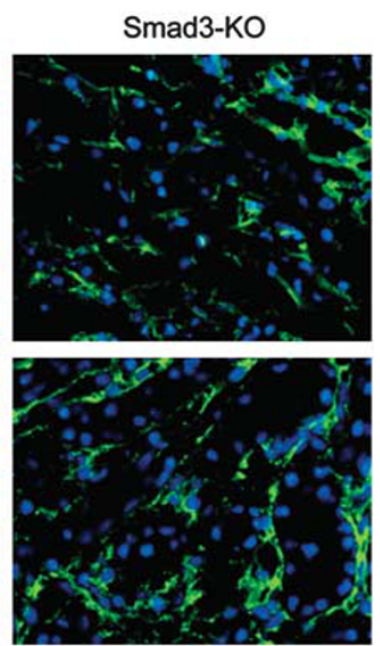

e

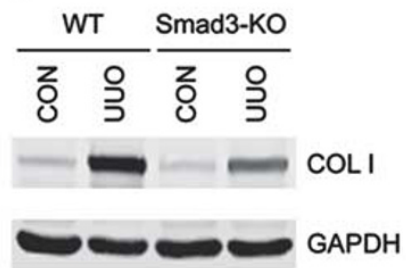

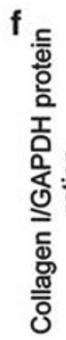

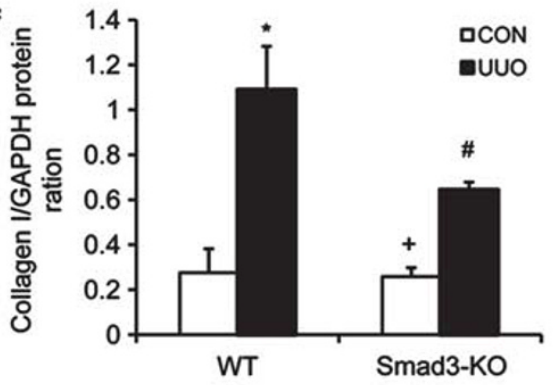

g

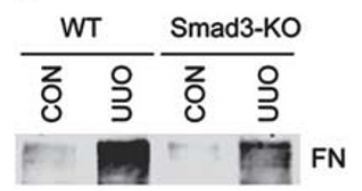

GAPDH h

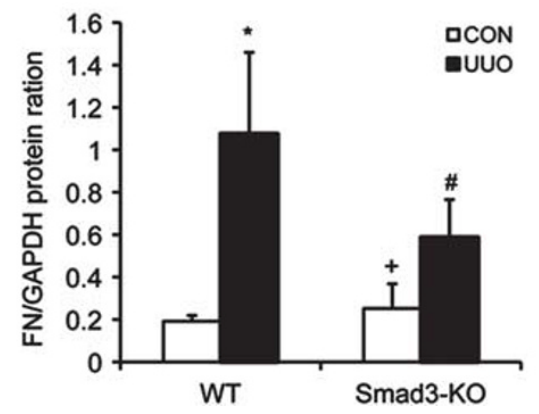

Figure 5 Smad3 deficiency inhibits collagen I and fibronectin expression in the kidney. (a) The mRNA levels of collagen I in the kidney of wild-type (WT) and Smad3-knockout (KO) mice as determined by real-time reverse transcription-polymerase chain reaction (RT-PCR). ${ }^{*} P<0.05$ vs WT controls, ${ }^{+} P<0.05$ vs KO unilateral ureteral obstruction (UUO), and ${ }^{\#} P<0.05$ vs WT UUO ( $n=4$ per group). (b) The mRNA levels of fibronectin in the kidney of WT and Smad3-KO mice as determined by real-time RT-PCR. ${ }^{*} P<0.05$ vs WT controls, ${ }^{+} P<0.05$ vs KO UUO, and ${ }^{\#} P<0.05$ vs WT UUO ( $n=4$ per group). (c) Representative photomicrographs of collagen I immunofluorescence staining in the kidney of WT and Smad3-KO mice at day 14 after UUO (original magnification: $\times 400)$. (d) Representative photomicrographs of fibronectin immunofluorescence staining in the kidney of WT and Smad3-KO mice at day 14 after UUO (original magnification: $\times$ 400). (e) Representative western blot analyses show the protein levels of collagen I in the kidney of WT and Smad3-KO mice. (f) Quantitative analysis of collagen I protein expression in the kidney of WT and Smad3-KO mice. ${ }^{*} P<0.05$ vs WT controls, ${ }^{+} P<0.05$ vs KO UUO, and ${ }^{\#} P<0.05$ vs WT UUO ( $n=4$ per group). (g) Representative western blot analyses show the protein levels of fibronectin in the kidney of WT and Smad3-KO mice. (h) Quantitative analysis of fibronectin protein expression in the kidney of WT and Smad3-KO mice. ${ }^{*} P<0.05$ vs WT controls, ${ }^{+} P<0.05$ vs KO UUO, and ${ }^{\#} P<0.05$ vs WT UUO ( $n=4$ per group). 
a
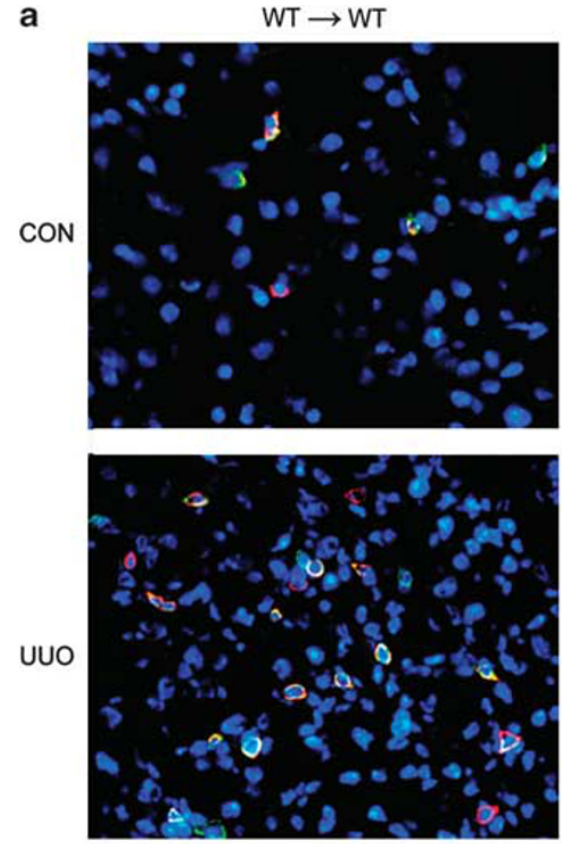

C
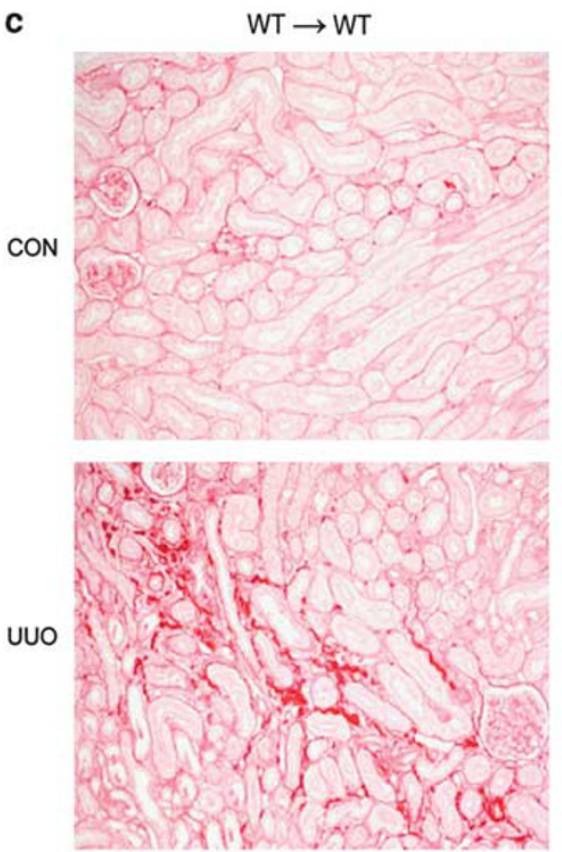
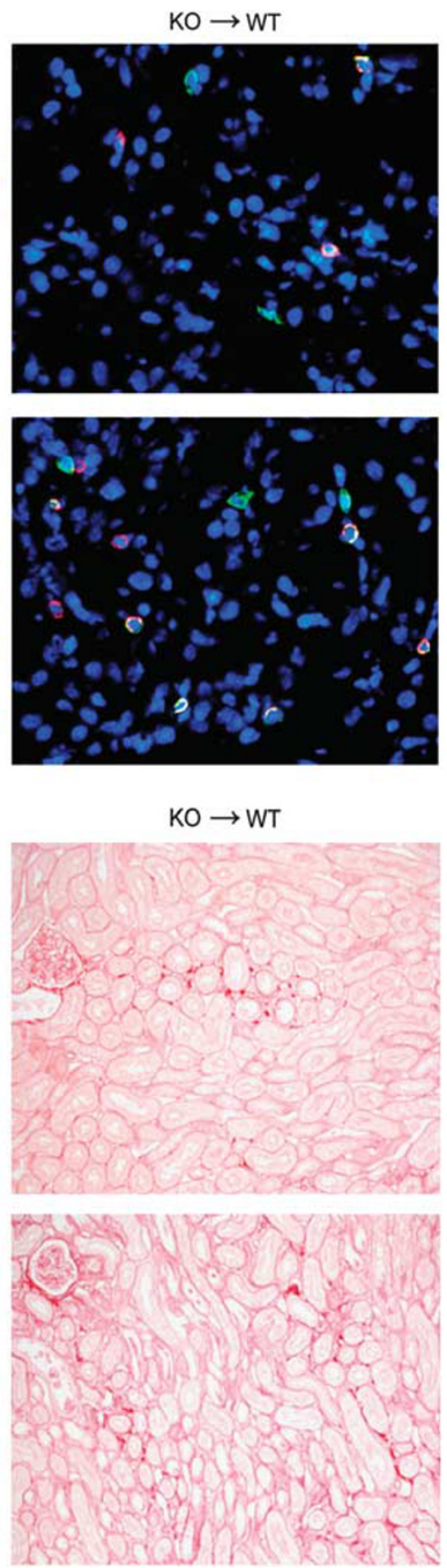
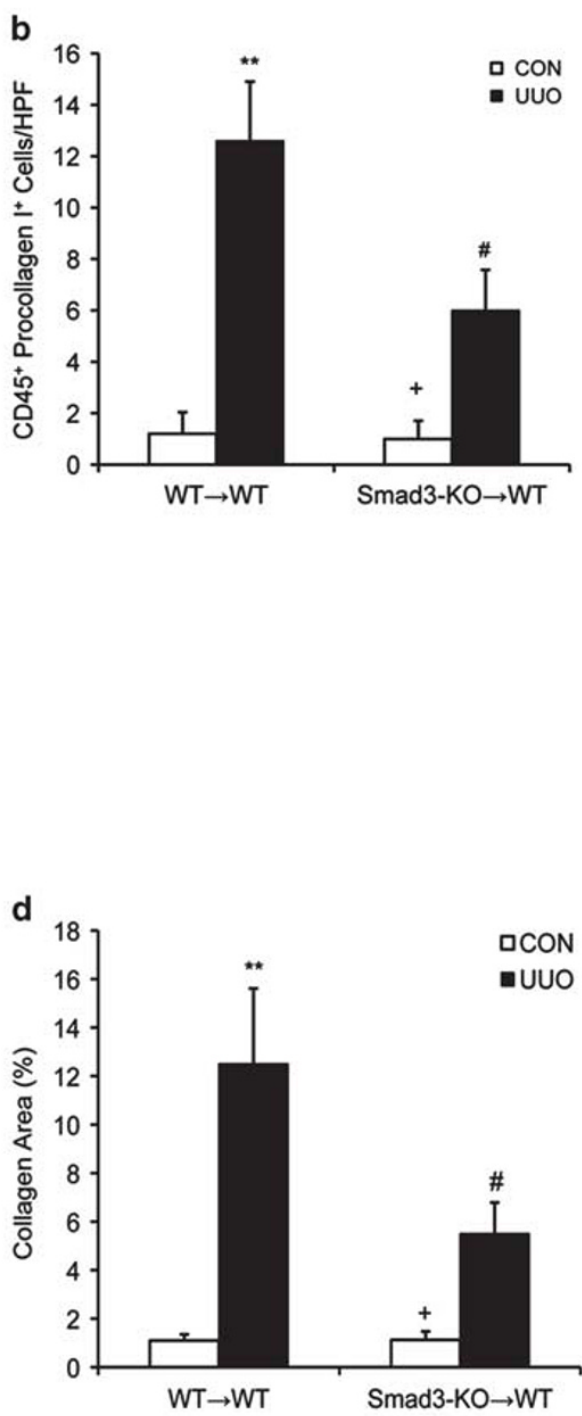

Figure 6 Smad3 deficiency in bone marrow-derived cells inhibits myeloid fibroblast accumulation and fibrosis. (a) Representative photomicrographs of kidney sections from wild-type (WT) mice transplanted with Smad3 $3^{+/+}$bone marrow cells and WT mice transplanted with Smad3 $3^{-1-}$ bone marrow cells 1 week after unilateral ureteral obstruction (UUO) stained for CD45 (red), procollagen I (green), and 4',6-diamidino-2-phenylindole (DAPI) (blue). (b) Quantitative analysis of $\mathrm{CD}_{4} 5^{+}$and procollagen I ${ }^{+}$fibroblasts in the kidney. ${ }^{* *} P<0.01$ vs WT $\rightarrow$ WT controls, ${ }^{+} P<0.05$ vs KO $\rightarrow$ WT UUO, and $\# P<0.05$ vs WT $\rightarrow$ WT UUO ( $n=5$ per group). (c) Representative photomicrographs show kidney sections stained with picrosirius red for assessment of total collagen deposition. (d) Quantitative analysis of renal interstitial collagen content in different groups as indicated. ${ }^{* *} P<0.01$ vs WT $\rightarrow$ WT controls, ${ }^{+} P<0.05$ vs KO $\rightarrow$ WT UUO, and ${ }^{\#} P<0.05$ vs WT $\rightarrow$ WT UUO ( $n=5$ per group).

\section{DISCUSSION}

Activated fibroblasts are responsible for wound healing and organ fibrosis. Recent studies have shown that bone marrowderived fibroblasts have a significant role in the pathogenesis of renal fibrosis. ${ }^{8}, 11,14,34$ The signaling mechanisms underlying the accumulation and activation of bone marrow- derived fibroblasts in the kidney are incompletely understood. We have previously shown that CXCL16 has an obligatory role in the recruitment of myeloid fibroblasts into the kidney and the development of renal fibrosis in response to obstructive injury. ${ }^{11}$ In the present study, we demonstrate that Smad3 signaling is pathologically 
a
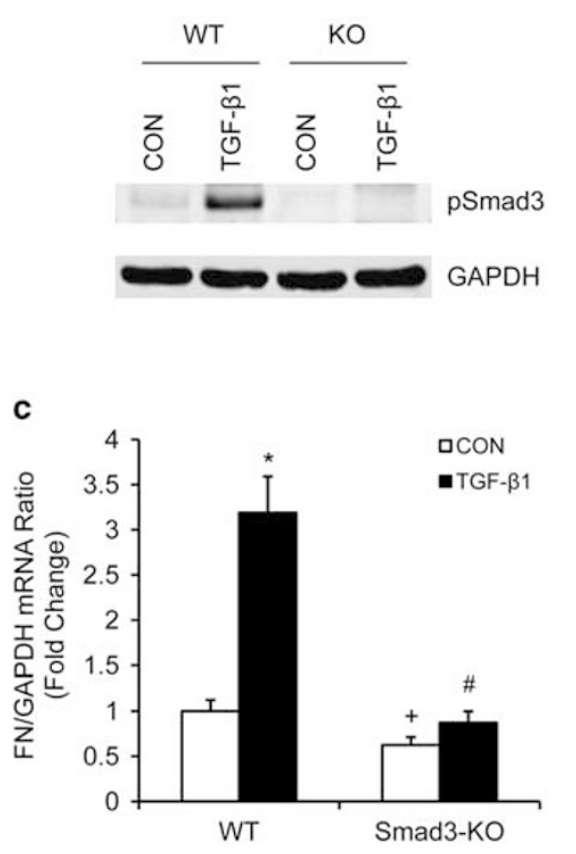
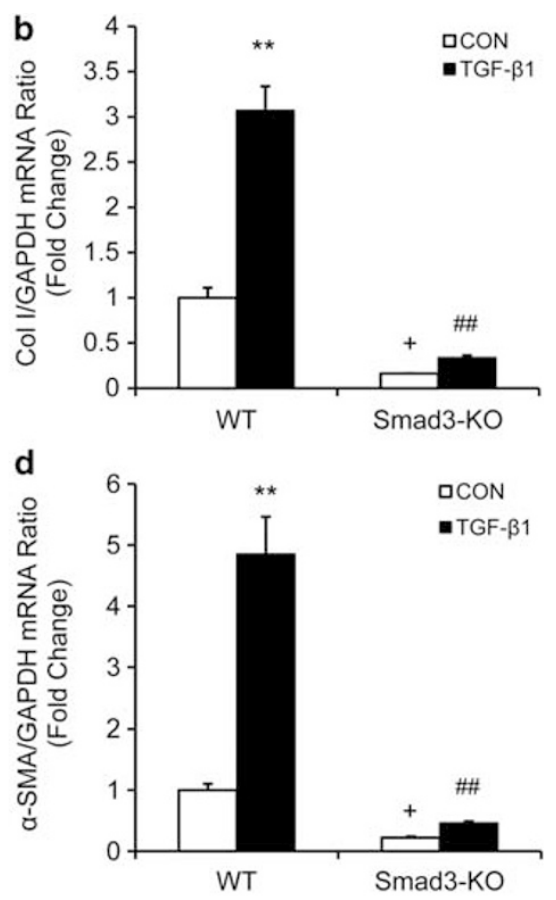

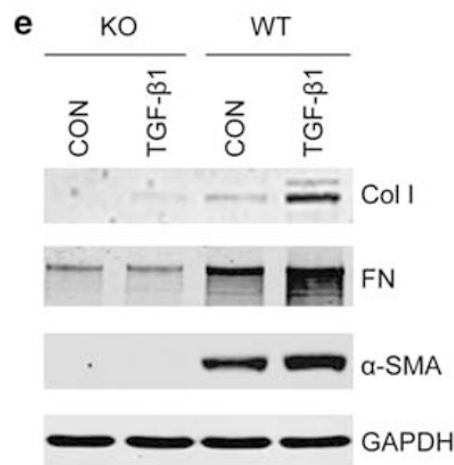

Figure 7 Smad3 deficiency inhibits tumor growth factor- $\beta 1$ (TGF- $\beta 1$ )-induced monocyte-to-fibroblast transition. (a) Representative western blot analyses show that Smad3 is activated in monocytes treated with TGF- $\beta 1$. (b) The mRNA levels of collagen I as determined by real-time reverse transcription-polymerase chain reaction (RT-PCR). ${ }^{*} P<0.05$ vs wild-type (WT) controls, ${ }^{+} P<0.05$ vs knockout (KO)-TGF- $\beta 1$, and ${ }^{\# \#} P<0.01$ vs WT-TGF- $\beta 1$ ( $n=4$ per group). (c) The mRNA levels of fibronectin as determined by real-time RT-PCR. ${ }^{*} P<0.05$ vs WT controls, ${ }^{+} P<0.05$ vs KO-TGF- $\beta 1$, and ${ }^{\#} P<0.05$ vs WT-TGF- $\beta 1$ ( $n=4$ per group). (d) The mRNA levels of $\alpha$-smooth muscle actin $\left(\alpha\right.$-SMA) as determined by real-time RT-PCR. ${ }^{* *} P<0.01$ vs WT controls, ${ }^{+} P<0.05$ vs KO-TGF- $\beta 1$, and ${ }^{\# \#} P<0.01$ vs WT-TGF- $\beta 1$ ( $n=4$ per group). (e). Representative western blot analyses show the protein levels of collagen I, fibronectin, and $\alpha$-SMA.

important in the activation of myeloid fibroblasts and the development of renal fibrosis because genetic disruption of Smad3 inhibits the activation of bone marrow-derived fibroblasts in the kidney in response to obstructive injury in vivo and suppresses monocyte-to-fibroblast transition in vitro. These data indicate that Smad3 signaling has an important role in the transformation of bone marrowderived fibroblasts in the kidney.

TGF- $\beta 1$ is a multifunctional cytokine that regulates ECM production by signaling through the type I and type II serine/ threonine kinase receptors. ${ }^{35}$ TGF- $\beta 1$ binds to receptor II and results in the phosphorylation of receptor I. The activated receptor I then directly signals to downstream intracellular substrates, the receptor-associated Smads (R-Smads), Smad2 and Smad3, through phosphorylation. Activated R-Smads heteroligomerize with the common Smad (Co-Smad), Smad4, and translocate into the nucleus to regulate target gene expression. ${ }^{36} \mathrm{~A}$ large body of evidence has shown that TGF- $\beta 1$ has a central role in the pathogenesis of renal fibrosis. ${ }^{20-22}$ Smad3 is a major downstream signaling molecule of TGF- $\beta 1$ in mediating the pathogenesis of fibrosis. ${ }^{27,37}$ In our study, we show that Smad3 signaling in myeloid fibroblasts is activated in response to obstructive injury in vivo. Genetic disruption of Smad3 suppressed the accumulation of bone marrow-derived fibroblasts in the kidney. These results indicate that Smad3 signaling has an important role in the activation of bone marrow-derived fibroblasts. 


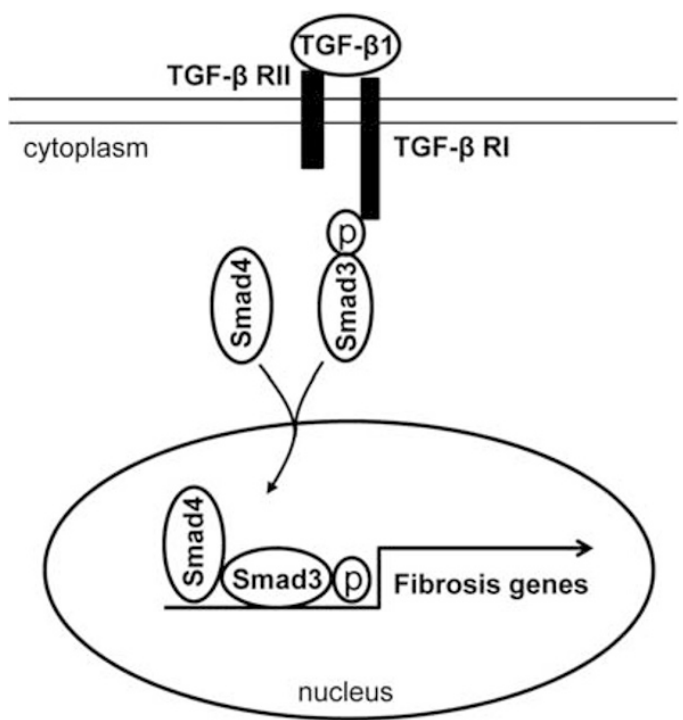

Figure 8 Schematic illustration of the signaling events leading to the activation of myeloid fibroblasts. Tumor growth factor- $\beta 1$ (TGF- $\beta 1$ ) binding to its receptor results in Smad3 phosphorylation. The phosphorylated Smad3 along with Smad4 is translocated into the nucleus, where it induces the expression of ECM genes and activation of myeloid fibroblasts.

Myofibroblasts are the active form of fibroblasts that are generally considered to be the main source of increased ECM deposition in renal fibrosis. ${ }^{4,5}$ Furthermore, experimental and clinical studies have shown that the number of interstitial myofibroblasts correlates closely with the severity of tubulointerstitial fibrosis and the progression of kidney disease. ${ }^{38-40}$ In the present study, we demonstrate that myofibroblasts identified as $\alpha$-SMA-positive cells accumulate in the kidney of WT mice following obstructive injury, and their accumulation is significantly reduced in the obstructed kidney of Smad3-KO mice. These results strongly indicate that Smad3 signaling activates bone marrow-derived myofibroblasts in the kidney.

A key feature of renal fibrosis is the marked increase and deposition of extracellular matrix proteins, including collagens and fibronectin. Morphometric analysis of picrosirius red staining of kidney sections at day 14 after obstructive injury demonstrates the presence of interstitial collagen deposition. This collagen deposition is significantly attenuated in the obstructed kidney of Smad3-KO mice. In keeping with these findings, we further illustrate that the expression of two major ECM proteins, collagen I and fibronectin, are markedly increased in the obstructed kidney of WT mice, whereas these responses are significantly attenuated in the injured kidney of Smad3-KO mice.

Although studies have shown that activation of Smad3 signaling has an important role in the pathogenesis of renal fibrosis, the cell types in which Smad3 is activated has not been clearly defined. One study claims that activation of Smad3 in tubular epithelial cells mediates renal fibrosis. ${ }^{27}$ Another study reports that $S \operatorname{mad} 2 / 3$ is activated in cells located in renal interstitial space. ${ }^{41}$ Using a phospho-Smad3specific antibody, our results show that Smad3 is activated in bone marrow-derived fibroblasts in the kidney in response to obstructive injury. Furthermore, the results of bone marrow chimeric experiments demonstrate that Smad3 signaling in bone marrow-derived fibroblasts contribute significantly to the pathogenesis of renal fibrosis following obstructive injury. The importance of Smad3 signaling in the activation of bone marrow-derived fibroblasts was further supported by our in vitro studies.

In summary, our study defines a novel mechanism by which Smad3 signaling regulates the pathogenesis of renal fibrosis. In response to injury, activated Smad3 signaling transforms $\mathrm{CD}_{4}{ }^{+}$myeloid fibroblasts into myofibroblasts, which contribute to the pathogenesis of renal fibrosis (Figure 8). These data suggest that Smad3 signaling in bone marrow-derived fibroblasts is essential for the development of renal fibrosis.

\section{ACKNOWLEDGMENTS}

We thank Dr William E Mitch for helpful discussion and Dr Xiao-Fan Wang for providing Smad3-KO mice. We also thank Joel M Sederstrom of the flow cytometry core at Baylor College of Medicine for technical assistance. This work was supported in part by the NIH grants (K08HL92958 and R01DK95835) and an American Heart Association grant (11BGIA7840054) to YW. The Cytometry and Cell Sorting Core at Baylor College of Medicine was supported in part by the NIH Grants (Al036211, CA125123, and RR024574).

\section{DISCLOSURE/CONFLICT OF INTEREST}

The authors declare no conflict of interest.

1. Schainuck LI, Striker GE, Cutler RE, et al. Structural-functional correlations in renal disease. II. The correlations. Hum Pathol 1970;1 631-641.

2. Nath KA. The tubulointerstitium in progressive renal disease. Kidney Int 1998:54:992-994.

3. Eddy AA. Molecular basis of renal fibrosis. Pediatr Nephrol 2000; 15:290-301.

4. Neilson EG. Mechanisms of disease: fibroblasts - a new look at an old problem. Nat Clin Pract Nephrol 2006;2:101-108.

5. Strutz F, Muller GA. Renal fibrosis and the origin of the renal fibroblast. Nephrol Dial Transplant 2006;21:3368-3370.

6. Iwano $M$, Plieth $D$, Danoff TM, et al. Evidence that fibroblasts derive from epithelium during tissue fibrosis. J Clin Invest 2002;110:341-350.

7. Li J, Deane JA, Campanale NV, et al. The contribution of bone marrowderived cells to the development of renal interstitial fibrosis. Stem Cells 2007:25:697-706.

8. Sakai $\mathrm{N}$, Wada $\mathrm{T}$, Yokoyama $\mathrm{H}$, et al. Secondary lymphoid tissue chemokine (SLC/CCL21)/CCR7 signaling regulates fibrocytes in renal fibrosis. Proc Natl Acad Sci USA 2006;103:14098-14103.

9. Grimm PC, Nickerson P, Jeffery J, et al. Neointimal and tubulointerstitial infiltration by recipient mesenchymal cells in chronic renalallograft rejection. N Engl J Med 2001;345:93-97.

10. Broekema M, Harmsen MC, van Luyn MJ, et al. Bone marrow-derived myofibroblasts contribute to the renal interstitial myofibroblast population and produce procollagen I after ischemia/reperfusion in rats. J Am Soc Nephrol 2007;18:165-175.

11. Chen G, Lin SC, Chen J, et al. CXCL16 recruits bone marrow-derived fibroblast precursors in renal fibrosis. J Am Soc Nephrol 2011;22: 1876-1886.

12. Yang J, Lin SC, Chen G, et al. Adiponectin promotes monocyte-tofibroblast transition in renal fibrosis. J Am Soc Nephrol 2013;24: 1644-1659. 
13. Bucala R, Spiegel LA, Chesney J, et al. Circulating fibrocytes define a new leukocyte subpopulation that mediates tissue repair. Mol Med 1994;1:71-81.

14. Niedermeier M, Reich B, Rodriguez Gomez M, et al. CD4 + T cells control the differentiation of Gr1 + monocytes into fibrocytes. Proc Natl Acad Sci USA 2009;106:17892-17897.

15. Shao DD, Suresh R, Vakil V, et al. Pivotal advance: Th-1 cytokines inhibit, and Th-2 cytokines promote fibrocyte differentiation. J Leukocyte Biol 2008;83:1323-1333.

16. Xia Y, Entman ML, Wang Y. Critical role of CXCL16 in hypertensive kidney injury and fibrosis. Hypertension 2013;62:1129-1137.

17. Metz CN. Fibrocytes: a unique cell population implicated in wound healing. Cell Mol Life Sci 2003;60:1342-1350.

18. Quan TE, Cowper S, Wu SP, et al. Circulating fibrocytes: collagensecreting cells of the peripheral blood. Int J Biochem Cell Biol 2004; 36:598-606.

19. Abe R, Donnelly SC, Peng $T$, et al. Peripheral blood fibrocytes: differentiation pathway and migration to wound sites. J Immunol 2001;166:7556-7562.

20. Border WA, Noble NA. Transforming growth factor beta in tissue fibrosis. N Engl J Med 1994;331:1286-1292.

21. Border WA, Okuda S, Languino LR, et al. Suppression of experimental glomerulonephritis by antiserum against transforming growth factor beta 1. Nature 1990;346:371-374.

22. Bottinger EP. Bitzer M. TGF-beta signaling in renal disease. J Am Soc Nephrol 2002;13:2600-2610.

23. Lan HY. Diverse roles of TGF-beta/Smads in renal fibrosis and inflammation. Int J Biol Sci 2011;7:1056-1067.

24. Latella G, Vetuschi A, Sferra $R$, et al. Targeted disruption of Smad3 confers resistance to the development of dimethylnitrosamineinduced hepatic fibrosis in mice. Liver Int 2009;29:997-1009.

25. Huang XR, Chung AC, Yang $F$, et al. Smad3 mediates cardiac inflammation and fibrosis in angiotensin II-induced hypertensive cardiac remodeling. Hypertension 2010;55:1165-1171.

26. Verrecchia F, Chu ML, Mauviel A. Identification of novel TGF-beta / Smad gene targets in dermal fibroblasts using a combined cDNA microarray/promoter transactivation approach. J Biol Chem 2001;276:17058-17062

27. Sato M, Muragaki Y, Saika S, et al. Targeted disruption of TGF-beta1/ Smad3 signaling protects against renal tubulointerstitial fibrosis induced by unilateral ureteral obstruction. J Clin Invest 2003;112: 1486-1494.

28. Zhao J, Shi W, Wang YL, et al. Smad3 deficiency attenuates bleomycininduced pulmonary fibrosis in mice. Am J Physiol Lung Cell Mol Physiol 2002;282:L585-L593.

29. Datto MB, Frederick JP, Pan L, et al. Targeted disruption of Smad3 reveals an essential role in transforming growth factor beta-mediated signal transduction. Mol Cell Biol 1999;19:2495-2504.

30. Yang J, Chen J, Yan J, et al. Effect of interleukin 6 deficiency on renal interstitial fibrosis. PLoS One 2012;7:e52415.

31. Xia Y, Entman ML, Wang Y. CCR2 regulates the uptake of bone marrowderived fibroblasts in renal fibrosis. PLoS One 2013;8:e77493.

32. Crawford JR, Pilling D, Gomer RH. Improved serum-free culture conditions for spleen-derived murine fibrocytes. J Immunol Methods 2010;363:9-20

33. Hong KM, Belperio JA, Keane MP, et al. Differentiation of human circulating fibrocytes as mediated by transforming growth factor-beta and peroxisome proliferator-activated receptor gamma. J Biol Chem 2007;282:22910-22920.

34. Lebleu VS, Taduri G, O'Connell J, et al. Origin and function of myofibroblasts in kidney fibrosis. Nat Med 2013;19:1047-1053.

35. Shi $Y$, Massague J. Mechanisms of TGF-beta signaling from cell membrane to the nucleus. Cell 2003;113:685-700.

36. Miyazono K. Positive and negative regulation of TGF-beta signaling. J Cell Sci 2000;113(Pt 7):1101-1109.

37. Roberts $A B$, Piek $E$, Bottinger EP, et al. Is Smad3 a major player in signal transduction pathways leading to fibrogenesis? Chest 2001;120 (Suppl):43S-47S

38. Zhang G, Moorhead PJ, el Nahas AM. Myofibroblasts and the progression of experimental glomerulonephritis. Exp Nephrol 1995; 3:308-318.

39. Roberts IS, Burrows C, Shanks $\mathrm{JH}$, et al. Interstitial myofibroblasts: predictors of progression in membranous nephropathy. J Clin Pathol 1997;50:123-127.

40. Essawy M, Soylemezoglu O, Muchaneta-Kubara EC, et al Myofibroblasts and the progression of diabetic nephropathy. Nephrol Dial Transplant 1997;12:43-50.

41. Inazaki K, Kanamaru Y, Kojima Y, et al. Smad3 deficiency attenuates renal fibrosis, inflammation, and apoptosis after unilateral ureteral obstruction. Kidney Int 2004;66:597-604. 